\title{
Deficiência de Vitamina D no Diabetes Mellitus: revisão integrativa da literatura
}

\author{
Vitamin D deficiency in Diabetes Mellitus: integrative literature review \\ Deficiencia de Vitamina D en Diabetes Mellitus: revisión integral de la literatura
}

Rafael Everton Assunção Ribeiro da Costa ${ }^{1 *}$, Márcia Rodrigues Coêlho², Manoel Pinheiro Lucio Neto ${ }^{2}$.

\section{RESUMO}

Objetivo: Analisar a influência da deficiência de Vitamina $D$ no desenvolvimento do diabetes mellitus. Métodos: Trata-se de uma revisão integrativa da literatura, ou de fonte secundária. Realizou-se levantamento bibliográfico de artigos nas bases de dados: Scientific Eletronic Library Online (SciELO), Bases de Dados da Literatura Latino Americana em Ciências de Saúde (LILACS), base de dados PubMed, da US National Library of Medicine e Google Acadêmico, no período de 2008 a 2018, com o uso dos descritores: "Vitamina D", "Diabetes Mellitus" e "Deficiência de Vitamina D". Resultados: A insuficiência de Vitamina D tem sido relatada em todo o mundo e em todos os grupos etários nos últimos anos e tem sido considerada um problema de saúde pública, uma vez que níveis reduzidos de Vitamina $\mathrm{D}$ se mostraram relacionados a várias doenças crônicas, como diabetes mellitus tipo 2, obesidade e hipertensão. A Vitamina $D$ está relacionada à homeostase e ao bom funcionamento do sistema imunológico. Considerações finais: Conclui-se que a Hipovitaminose D é um achado constante em pacientes que apresentam diabetes mellitus e que essa relação é inversamente proporcional ao tempo e ao agravamento da doença.

Palavras-chave: Vitamina D, Diabetes Mellitus, Deficiência de Vitamina D.

\section{ABSTRACT}

Objective: To analyze the influence of Vitamin D deficiency on the development of diabetes mellitus. Methods: It is an integrative literature review study, or by secondary source. A bibliographical survey was carried out, through queries to articles in the databases: Scientific Electronic Library Online (SciELO), Databases of the Latin American Literature in Health Sciences (LILACS), US National Library of Medicine database - PubMEd and Google Scholar, in the period of 2008 to 2018, by mean of the descriptors: "Vitamin D", "Diabetes Mellitus" and "Vitamin D Deficiency". Results: The results showed that Vitamin D insufficiency has been reported worldwide in all age groups in recent years. It has been considered a public health problem, since the decrease in Vitamin D levels has been related to several chronic diseases, such as diabetes mellitus type 2, obesity, and hypertension. Vitamin D is related to homeostasis and the proper functioning of the immune system. Final considerations: We conclude that Vitamin D deficiency is a constant finding in patients with diabetes mellitus and that this relationship is inversely proportional to time and aggravation of the disease.

Keywords: Vitamin D, Diabetes Mellitus, Vitamin D deficiency.

\section{RESUMEN}

Objetivo: Analizar la influencia de la deficiencia de Vitamina $D$ en el desarrollo de la diabetes mellitus. Métodos: Esta es una revisión integral de la literatura, o una de fuente secundaria. Se realizó una encuesta bibliográfica de artículos en las bases de datos: Scientific Eletronic Library Online (SciELO), Bases de Datos de Literatura Latinoamericana en Ciencias de la Salud (LILACS), PubMed database, de la Biblioteca Nacional de Medicina de EE. UU. y Google Académico, de 2008 a 2018, utilizando los descriptores: "Vitamina D", "Diabetes Mellitus" y "Deficiencia de Vitamina D". Resultados: La insuficiencia de Vitamina D se ha informado en todo el mundo y en todos los grupos de edad en los últimos años y se ha considerado un problema de salud pública, ya que se ha demostrado que los niveles reducidos de Vitamina D están relacionados con varias enfermedades crónicas, tales como diabetes mellitus tipo 2, obesidad e hipertensión. La Vitamina D está relacionada con la homeostasis y el buen funcionamiento del sistema inmunitario. Consideraciones finales: Concluimos que la Hipovitaminosis D es un hallazgo constante en pacientes con diabetes mellitus y que esta relación es inversamente proporcional al tiempo y al empeoramiento de la enfermedad.

Palabras clave: Vitamina D, Diabetes Mellitus, Deficiencia de Vitamina D.

${ }^{1}$ Universidade Estadual do Piauí (UESPI), Teresina - PI. *E-mail: rafassuncao.rafael@gmail.com

${ }^{2}$ Centro Universitário Santo Agostinho (UNIFSA), Teresina - PI. 


\section{INTRODUÇÃO}

A Vitamina D3 (colecalciferol) é considerada um hormônio, que é sintetizado nos tecidos da pele por meio de uma isomerização acelerada pela radiação ultravioleta (UV), de comprimentos de onda entre 290 a 315 $\mathrm{nm}$. Essa vitamina é uma importante reguladora do metabolismo do fósforo e do cálcio, bem como participa da formação óssea e tem sido objeto de estudo crescente na investigação médica (LOPES PP, 2014; RAPURI PB, et al., 2002).

A deficiência de Vitamina $D$ está associada a uma menor mineralização do tecido osteóide, caracterizando a osteomalácia (PFEIFER M, et al., 2002; ROBINS SP e NEW SA, 1997). Entretanto, análises mais recentes demonstram que valores não tão reduzidos de Vitamina $D$ estão associados a aumento do "turn over" ósseo e consequente aumento de fraturas.

A Hipovitaminose D é bastante presente na população geral e impacta na saúde pública global. A literatura aponta uma alta prevalência dessa patologia, que é distribuída em variadas áreas do planeta, incluindo o Brasil, chegando a estar presente em cerca de $90 \%$ da população em alguns locais (MITHAL A, et al., 2009).

O RDA (Recommended Dietary Allowance) acusa a necessidade de ingestão de $400 \mathrm{UI}$ de Vitamina D por dia para pessoas entre 50 e 70 anos e de $600 \mathrm{UI}$ para aqueles com mais de 70 anos, o que também pode ser conseguido com 20 minutos de exposição diária de $5 \%$ da superfície corpórea ao sol. Os alimentos que apresentam Vitamina $\mathrm{D}$, como peixes de águas frias e cogumelos, possuem baixa quantidade desta e não são usualmente ingeridos pela população geral (SARAIVA GL, et al., 2007).

O diabetes é um grupo de doenças metabólicas caracterizado por hiperglicemia resultante de defeitos na secreção de insulina, ação da insulina ou ambos. O aumento sérico de glicose que ocorre no diabetes mellitus costuma cursar com lesão de órgãos-alvo (olhos, rins e sistemas nervoso e cardiovascular) ao longo do tempo. Vários processos patogênicos estão envolvidos no desenvolvimento do diabetes. Estes variam desde a destruição autoimune das células $\beta$ do pâncreas, com consequente deficiência de insulina, até anormalidades que resultam em resistência à ação da insulina (BOILAT-BLANCO N, et al., 2016).

Diversos estudos realizados na área correlacionam a Hipovitaminose $D$ à ocorrência de inflamação e agravamento do diabetes mellitus tipo 1 e 2 (DM1 e 2). Pesquisas in vivo apontam a Vitamina $D$ como um fator de proteção a estas patologias (PITTAS AG, et al., 2007).

Alguns estudos têm evidenciado uma relação entre a diabetes e os níveis inadequados de Vitamina D. A função clássica da Vitamina $D$ na saúde óssea já é bem conhecida, porém evidências recentes sugerem que esta vitamina pode ter funções extra-esqueléticas importantes na redução do risco de várias outras doenças, incluindo as doenças crônicas. A deficiência de Vitamina $D$ constitui, neste caso, um fator de risco não clássico (ZITTERMAN A e GUMMERT JF, 2010; HOLICK MF, 2007).

Mais estudos terão de ser realizados sobre a relação entre a deficiência de Vitamina $D$ e o diabetes mellitus e, se for confirmado que esta relação é causal, intervir nessa deficiência da população, com suplementação e/ou fortificação de Vitamina D. Tendo em conta as recomendações, a exposição solar pode ser um meio simples e potencial para abrandar o desenvolvimento de doenças crônicas, como o diabetes, na população em geral. Diante disso, a presente pesquisa teve por objetivo analisar a influência da deficiência de Vitamina $\mathrm{D}$ no desenvolvimento do diabetes mellitus.

\section{MÉTODOS}

Trata-se de um estudo de revisão integrativa da literatura, ou de fonte secundária. Segundo Cervo AL, et al. (2007), a revisão integrativa da literatura busca tomar conhecimento e fazer uma avaliação das colaborações científicas no passado acerca de um determinado assunto, tema ou problema.

O levantamento bibliográfico foi realizado no período de Janeiro a Março de 2018, por intermédio de consultas a artigos nas bases de dados: Scientific Eletronic Library Online (SciELO), Bases de dados da literatura Latino Americana em Ciências de Saúde (LILACS), Base de Dados PubMed, da US National Library of Medicine e Google acadêmico, no período de 2008 à 2018, por meio dos descritores: "Vitamina D", "Diabetes Mellitus" e "Deficiência de Vitamina D", confirmados nos Descritores em Ciência da Saúde (DeCS). 
Utilizou-se como critérios de inclusão: artigos dentro da faixa de anos especificada (2008 a 2018), com texto completo disponível para leitura e que forneciam informações suficientes ao tema do estudo. Adotou-se como critério de exclusão todos os parâmetros que não se adequaram aos critérios de inclusão estabelecidos. A análise foi realizada por meio de leitura e estudo compreensivo dos artigos; depois, foi realizada uma caracterização e, em seguida, categorização dos mesmos, com posterior leitura exploratória e abrangente. Os resultados foram agrupados em tabelas do Office Word 2013, para uma melhor discussão dos dados.

\section{RESULTADOS}

Este estudo se propôs a realizar um levantamento de estudos epidemiológicos nacionais sobre diabetes mellitus e sobre os cuidados prestados aos sujeitos diabéticos na Atenção Primária à Saúde (APS) no Brasil. Foi encontrado um total de 50 artigos por meio dos descritores utilizados.

No decorrer da pesquisa, houve referências que não se relacionavam com a temática. Realizou-se uma análise minuciosa e apenas 30 artigos se adequaram aos critérios de inclusão, sendo excluídos, dessa forma, 20 artigos (Figura 1). Neste sentido, foi encontrado 01 artigo no ano de 2009, 2 artigos no ano de 2010, 4 artigos no ano de 2013, 5 artigos no ano de 2014, 7 artigos no ano de 2015, 6 artigos no ano de 2016 e 5 artigos no ano de 2017. A Figura 2 representa a porcentagem de artigos encontrada por ordem cronológica.

Figura 1 - Artigos selecionados de acordo com os critérios estabelecidos.

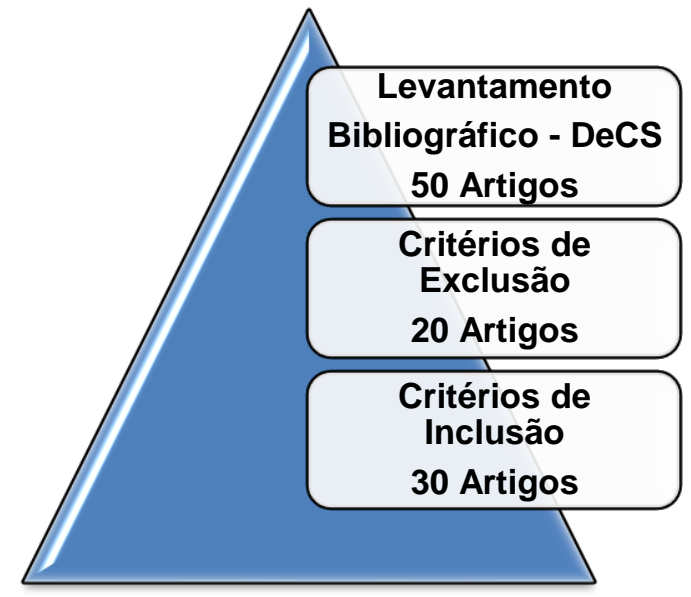

Fonte: Costa REAR, et al., 2018.

Figura 2 - Porcentagem de artigos de acordo com o ano de publicação.

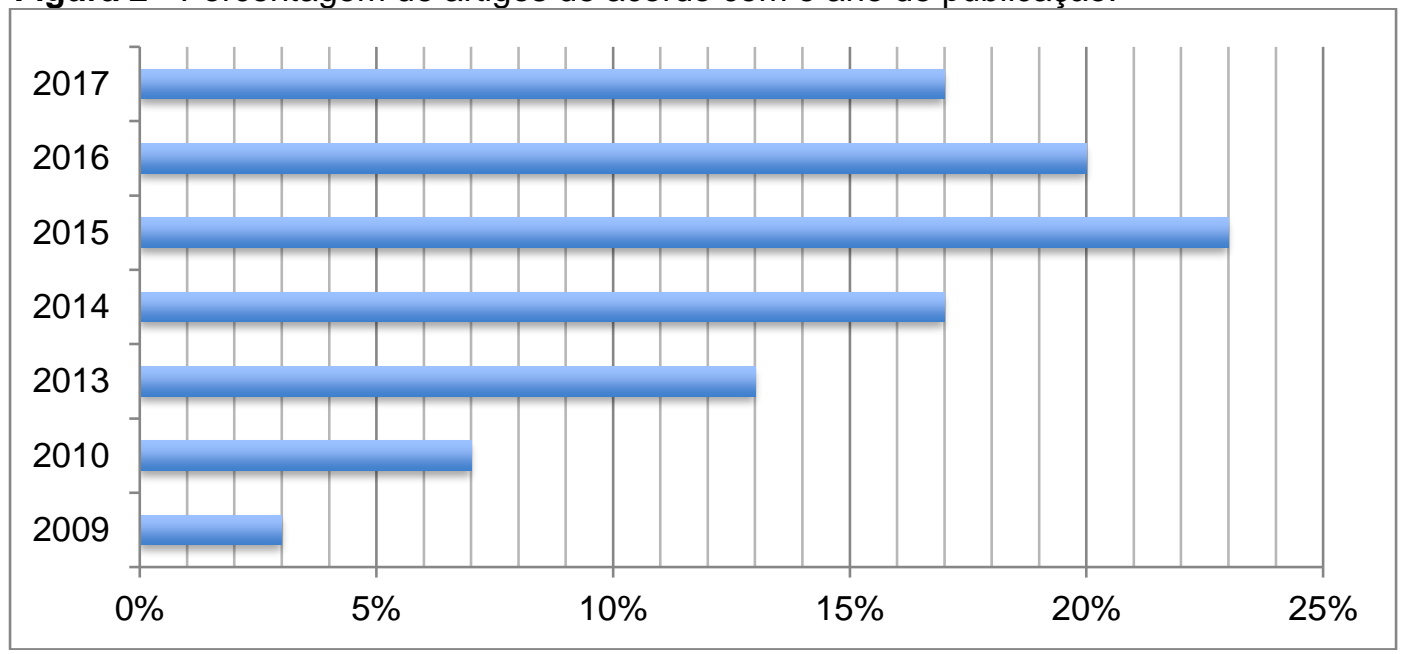

Fonte: Costa REAR, et al., 2018.

A presente revisão lida com estudos relacionados à deficiência de Vitamina $\mathrm{D}$ com o diabetes mellitus. No Quadro 1, estão apresentados os 30 artigos encontrados nas bases de pesquisa. Nele, estão contidos os títulos dos artigos, os autores, as revistas de publicação e o ano em que foram publicados. 


\section{Revista Eletrônica Acervo Saúde / Electronic Journal Collection Health ｜ ISSN 2178-2091}

Quadro 1 - Artigos referentes à deficiência de Vitamina $D$ e o diabetes mellitus.

\begin{tabular}{|c|c|c|c|}
\hline Título & Autores & Revista & Ano \\
\hline Vitamina D e doenças endócrino metabólicas & Schuch NJ, et al. & Arquivos Brasileiros de Endocrinologia \& Metabologia & 2009 \\
\hline Ações não esqueléticas baseadas em evidência da Vitamina D & Muszkat $P$, et al. & Arquivos Brasileiros de Endocrinologia \& Metabologia & 2010 \\
\hline A importância dos níveis de Vitamina D nas doenças autoimunes & Marques CDL, et al. & Revista Brasileira de Reumatologia & 2010 \\
\hline Vitamina D: ações extraósseas e uso racional & Lichtenstein A, et al. & Revista da Associação Médica Brasileira & 2013 \\
\hline Vitamina D e controle glicêmico no diabetes mellitus tipo 2 & Kostoglou-athanassiou I, et al. & $\begin{array}{l}\text { Therapeutic Advances in Endocrinology and } \\
\text { Metabolism }\end{array}$ & 2013 \\
\hline $\begin{array}{c}\text { Deficiência de Vitamina } \mathrm{D} \text { em pacientes com diabetes mellitus tipo } 2 \\
\text { na região sul da Arábia Saudita }\end{array}$ & Alhumaidi M, et al. & Journal of Clinical Medicine & 2013 \\
\hline $\begin{array}{c}25(\mathrm{OH}) \text { D sérica no diabetes mellitus tipo 2: relação com o controle } \\
\text { glicêmico }\end{array}$ & Dhia A e Ali A & Journal of Clinical and Diagnostic Research & 2013 \\
\hline $\begin{array}{l}\text { Vitamina D e diabetes mellitus, suas epidemias e o envelhecimento. } \\
\text { O que há de novo? }\end{array}$ & Machado MRC, et al. & Reprodução \& Climatério & 2014 \\
\hline Vitamina D e diabetes mellitus: uma atualização - 2013 & Griz LH, et al. & Arquivos Brasileiros de Endocrinologia \& Metabologia & 2014 \\
\hline $\begin{array}{c}\text { Efeitos da suplementação de Vitamina D na resistência à insulina e } \\
\text { fatores de risco cardiometabólicos em crianças com síndrome } \\
\text { metabólica: um ensaio controlado triplo-cego }\end{array}$ & Kelishadi R, et al. & Jornal de Pediatria & 2014 \\
\hline $\begin{array}{l}\text { Um ensaio prospectivo, randomizado e controlado dos efeitos da } \\
\text { suplementação de Vitamina } D \text { no controle glicêmico à longo prazo no } \\
\text { diabetes mellitus tipo } 2 \text { da Coréia }\end{array}$ & Ryu OH, et al. & EndocrineJournal & 2014 \\
\hline $\begin{array}{c}\text { Eficácia da suplementação de Vitamina D na melhoria dos } \\
\text { parâmetros glicêmicos em pacientes com diabetes mellitus tipo 2; um } \\
\text { ensaio clínico randomizado, duplo-cego }\end{array}$ & Nasri H, et al. & Jornal of Renal Injury Prevention & 2014 \\
\hline Influência da Vitamina D nas doenças endocrinometabólicas & Rafaelli RA, et al. & Semina: Ciências Biológicas e da Saúde & 2015 \\
\hline $\begin{array}{l}\text { Níveis séricos de Vitamina D3 em mulheres com diabetes mellitus } \\
\text { tipo } 2\end{array}$ & Cornacini $\mathrm{M}$, et al. & Journal of the Health Sciences Institute & 2015 \\
\hline $\begin{array}{l}\text { Não associação entre os níveis de 25-Hidroxivitamina D e pré- } \\
\text { diabetes em pacientes brasileiros. Um estudo transversal }\end{array}$ & Giorelli GO, et al. & Medical Journal & 2015 \\
\hline $\begin{array}{l}\text { A deficiência de Vitamina } D \text { está associada à presença e gravidade } \\
\text { da retinopatia diabética no diabetes mellitus tipo } 2 ?\end{array}$ & Alcublerre $\mathrm{N}$, et al. & Journal of Diabetes Reseaech & 2015 \\
\hline A Vitamina D desempenha um papel significativo no diabetes tipo 2? & Sheth JJ, et al. & BMC Endocrine Disorders & 2015 \\
\hline
\end{tabular}

REAS/EJCH | Vol.Sup.n.57 | e3994 | DOI: https://doi.org/10.25248/reas.e3994.2020 Página 4 de 9 


\section{Revista Eletrônica Acervo Saúde / Electronic Journal Collection Health ｜ ISSN 2178-2091}

\begin{tabular}{|c|c|c|c|}
\hline Título & Autores & Revista & Ano \\
\hline $\begin{array}{c}\text { A deficiência de Vitamina } \mathrm{D} \text { está relacionada à acumulação de } \\
\text { produtos finais de glicação avançada, marcadores de inflamação e } \\
\text { estresse oxidativo em pacientes diabéticos? }\end{array}$ & Šebeková K, et al. & BioMedResearchInternational & 2015 \\
\hline $\begin{array}{l}\text { Existe uma relação entre a Vitamina } \mathrm{D} \text { e a resistência à insulina e o } \\
\text { diabetes mellitus? }\end{array}$ & Al-Shoumer K e Al-Essa T & World Journal of Diabetes & 2015 \\
\hline Hipovitaminose D e desenvolvimento da síndrome metabólica & Oliveira B e Zeni S & Acta Bioquímica Clínica Latinoamericana & 2016 \\
\hline $\begin{array}{c}\text { Deficiência de Vitamina } D \text { em relação ao risco de síndrome } \\
\text { metabólica em pacientes de meia-idade e idosos com diabetes } \\
\text { mellitus tipo } 2\end{array}$ & Pan GT, et al. & J NutrSciVitaminol & 2016 \\
\hline $\begin{array}{c}\text { Associação de deficiência de Vitamina } \mathrm{D} \text { com controle glicêmico } \\
\text { deficiente em pacientes diabéticos }\end{array}$ & lqbal $\mathrm{K}$, et al. & J Pak MedAssoc & 2016 \\
\hline $\begin{array}{c}\text { Deficiência de Vitamina } D \text { e status glicêmicos em crianças e } \\
\text { adolescentes com diabetes mellitus tipo } 1\end{array}$ & Savastio S, et al. & PLOS One & 2016 \\
\hline $\begin{array}{c}\text { Associação entre nível sérico de Vitamina D e marcadores } \\
\text { glicêmicos e inflamatórios em pacientes não obesos com diabetes } \\
\text { tipo } 2\end{array}$ & Haidari $F$, et al. & Iran J MedSci & 2016 \\
\hline $\begin{array}{c}\text { Um ensaio controlado e randomizado avaliando o impacto da } \\
\text { suplementação direcionada de Vitamina D na função endotelial no } \\
\text { diabetes mellitus tipo 2: o ensaio DIMENSION }\end{array}$ & Dalan $R$, et al. & Diabetes \& Vascular DiseaseResearch & 2016 \\
\hline $\begin{array}{c}\text { Adipocinas séricas e níveis de Vitamina } \mathrm{D} \text { em pacientes com } \\
\text { diabetes mellitus tipo } 1\end{array}$ & Ismail MM, et al. & ArchMedSci & 2017 \\
\hline $\begin{array}{l}\text { Status da Vitamina D e sua associação com a resistência insuliníca } \\
\text { entre diabéticos tipo } 2 \text { : um estudo caso-controle no Gana }\end{array}$ & Fondjo LA, et al. & PLOS One & 2017 \\
\hline $\begin{array}{l}\text { Associação entre deficiência de Vitamina } \mathrm{D} \text { e retinopatia diabética no } \\
\text { diabetes tipo 2: uma metanálise de estudos observacionais }\end{array}$ & Luo B, et al. & Nutrients & 2017 \\
\hline $\begin{array}{l}\text { Associação entre } 25 \text {-Hidroxivitamina } \mathrm{D} \text { sérica e placa aterosclerótica } \\
\text { carotídea em pacientes chineses com diabetes tipo } 2\end{array}$ & Ding YH, et al. & Medicine & 2017 \\
\hline $\begin{array}{c}\text { Associação entre nível de Vitamina D e neuropatia periférica } \\
\text { diabética em pacientes com diabetes mellitus tipo 2: revisão } \\
\text { sistemática atualizada e metanálise }\end{array}$ & Qu GB, et al. & Journal of Clinical \& Translational Endocrinology & 2017 \\
\hline
\end{tabular}

Fonte: Costa REAR, et al., 2018. 


\section{DISCUSSÃO}

De acordo com os artigos encontrados, a insuficiência de Vitamina $D$ tem sido relatada em todo o mundo e em todos os grupos etários nos últimos anos e é considerada um problema de saúde pública, uma vez que a redução dos níveis de Vitamina $D$ tem sido relacionada a várias doenças crônicas, como diabetes mellitus tipo 2 (DM2) (CORNACINI M, et al., 2015), obesidade e hipertensão (KIMBALL S, et al., 2008).

A deficiência do metabolismo da glicose é uma das principais características do diabetes mellitus, o que pode levar a um quadro de hiperglicemia crônica. A doença pode se apresentar nas formas de diabetes tipo 1, tipo 2, gestacional e outros tipos específicos. O diabetes mellitus tipo 1 surge a partir da destruição das células beta do pâncreas, muitas vezes resultado de doenças autoimunes, gerando um quadro de insuficiência total de insulina. Nesses casos, os indivíduos necessitam serem tratados com insulina, evitando situações como cetoacidose, coma ou morte.

Outro tipo de diabetes mellitus, o tipo 2, é caracterizado pela resistência à ação da insulina e a carência desse hormônio gera incapacidade de compensar essa resistência (OLIVEIRA RC, 2006). Os demais tipos de diabetes mellitus são menos frequentes e podem derivar desde defeitos genéticos da função das células beta e da ação da insulina a doenças no pâncreas exócrino, infecções e efeito colateral de medicamentos, entre outros (PARISI MCR, 2003).

Segundo Schuch NJ, et al. (2009), a intolerância à glicose e à secreção de insulina foram observadas durante a deficiência de Vitamina $\mathrm{D}$, em modelos animais e humanos, resultando em DM2. O suposto mecanismo para esses achados é a apresentação do receptor de Vitamina $D$ em vários tecidos e células, incluindo células $\beta$-pancreáticas, adipócitos e células musculares (MUSZKAT P, et al., 2010).

Em indivíduos obesos, o comprometimento do sistema endócrino de Vitamina $\mathrm{D}$, caracterizado por níveis elevados de paratormônio (PTH) e 1,25 (OH) 2D3 (hormônio principal que atua como ligante para o fator de transcrição nuclear receptor da Vitamina $D$, regulando a transcrição gênica e a função celular em diversos tecidos), que poderia gerar um feedback negativo para a produção hepática de Vitamina $D$ e também cooperar para o aumento de cálcio intracelular, o que, por sua vez, leva à secreção de menos insulina e deteriora a sensibilidade à esta (RAFAELLI RA, et al., 2015; SCHUCH NJ, et al., 2009). Portanto, a Vitamina D é essencial para liberação de insulina, enquanto a suplementação de Vitamina $D$ restaura a secreção deste hormônio (GEDIK A e AKALIN S, 1986).

Para Muszkat $P$, et al. (2010), além de suas ações tradicionais relacionadas ao cálcio, a Vitamina $D$ e seus análogos sintéticos estão sendo cada vez mais reconhecidos por suas atividades anti-proliferativas, pródiferenciativas e imunomoduladoras. De acordo com os autores, a deficiência de Vitamina $D$ tem sido associada a um risco aumentado de diabetes mellitus tipo 1 (DMT1) e tipo 2 (PAN GT, et al., 2016), bem como o controle glicêmico e a resistência insulina são melhoradas quando a deficiência de Vitamina $D$ é corrigida, juntamente com a suplementação de cálcio (OLIVERI B e ZENI S, 2016).

Encontra-se na literatura uma relação entre a Vitamina $D$ e uma função adequada do sistema imune, tanto referente à imunidade inata quanto à imunidade adquirida (MARQUES CDL, et al., 2010), bem como com a regulação do tônus do Fator de Necrose Tumoral a (TNF-a) (HAIDARI F, et al., 2016). Assim, há evidências de que a deficiência de Vitamina $D$ implique em algumas doenças de cunho imunológico (diabetes mellitus, artrite reumatoide e lúpus eritematoso sistêmico, por exemplo), posto que tem se demonstrado que a regulação dos níveis de Vitamina $D$ implica em melhoria de parâmetros destas doenças em modelos experimentais. Lichtenstein A, et al. (2013) aponta uma crescente solicitação de exames laboratoriais no mundo, dentre os quais, pode-se destacar a dosagem sérica de Vitamina D. Estudos associam este fenômeno a um crescente números de trabalhos sobre o papel da Vitamina $D$ no organismo e sua relação com diversas patologias (THACHER TD e CLARKE BL, 2011).

Estudos realizados por Kostoglou-Athanassiou I, et al. (2013) revelaram que os níveis de $25(\mathrm{OH})$ D3 foram menores nos pacientes com diabetes mellitus tipo 2 do que no grupo controle (indivíduos saudáveis), sendo $19,26 \pm 0,95 \mathrm{ng} / \mathrm{ml}$ e $25,49 \pm 1,02 \mathrm{ng} / \mathrm{ml}$ nos grupos de pacientes e controle, respectivamente $(p<0,001$, Teste $t$ de Student). Os pesquisadores verificaram que os níveis de $25(\mathrm{OH})$ D3 estavam inversamente associados aos níveis de hemoglobina glicada ( $\mathrm{HbA1c}$ ) nos pacientes diabéticos. Verificou-se que os níveis 
de $25(\mathrm{OH})$ D3 estavam inversamente associados à $\mathrm{HbA1c}$ quando os grupos de pacientes e de controle foram analisados em conjunto. Os autores concluíram que os níveis de Vitamina D pareciam ser menores em pacientes com diabetes mellitus tipo 2 do que no grupo controle, estando os níveis de Vitamina $D$ relacionados ao controle glicêmico no diabetes mellitus tipo 2, sugerindo mais uma vez que a suplementação cautelosa de Vitamina $\mathrm{D}$ pode melhorar o controle glicêmico em diabetes mellitus tipo 2.

Esses achados corroboram como os estudos de Dhia A e Ali A (2013), que identificaram que um baixo nível de Vitamina $D$ está presente em dois terços dos pacientes com DM tipo 2, particularmente entre diabéticos com baixo controle glicêmico e entre aqueles com mais de 5 anos de diabetes (DHIA A e ALI A, 2013). O Diabetes Mellitus apresenta sintomas, como alterações na visão, fadiga, poliúria (excreção excessiva de urina), polidipsia (sede excessiva), fome constante e retinopatia, tais sintomas podem ocorrer repentinamente (OLIVEIRA KC e ZANETTI ML, 2011). Um estudo realizado por Alcublerre N, et al. (2015), com o objetivo de averiguar se há uma associação de status de Vitamina $D$ e retinopatia diabética no diabetes tipo 2, confirmou a associação da deficiência de Vitamina $D$ com a presença e gravidade da retinopatia diabética no diabetes tipo 2.

Apesar dos estudos apontarem que indivíduos diabéticos apresentam Hipovitaminose D, Alhumaidi M, et al. (2013), por meio de pesquisas sobre os índices de Vitamina $D$ em pessoas saudáveis e diabéticas na Arábia Saudita, constatou que os níveis séricos médios de Vitamina $D$ no grupo diabético foram de 15,7 + 7,5 $\mathrm{ng} / \mathrm{mL}$ em comparação ao grupo não diabético saudável com 11,1 +5,9 ng / mL, em um total de 340 pacientes. Esses resultados sugeriram que $98,5 \%$ dos dois grupos estudados tinham deficiência em Vitamina $D$, indicando a necessidade de suplementação de Vitamina D também em indivíduos saudáveis.

Para Machado MRC, et al. (2014), a incidência da deficiência de Vitamina D tem sido relatada até mesmo no Brasil, que é uma região ensolarada. Em Recife (latitude $10^{\circ} \mathrm{S}$ ), a prevalência de deficiência de Vitamina $\mathrm{D}$ em mulheres pós-menopausa foi de $8 \%$ para valores abaixo de $15 \mathrm{ng} / \mathrm{mL}$ e $43 \%$ para aqueles abaixo de 25 $\mathrm{ng} / \mathrm{mL}$. Já na Itália, em estudo observacional feito em mulheres também no período de pós-menopausa, os níveis de Vitamina $\mathrm{D}$ foram menores nas pacientes portadoras de DM2 do que no grupo controle, 39\% versus 25\% (GRIZ LH, et al., 2014).

Kelishadi $\mathrm{R}$, et al. (2014) realizou um estudo com 50 crianças e adolescentes, que foram separados em dois grupos (caso e controle). Durante 12 semanas, o grupo caso recebeu Vitamina D oral em $300.000 \mathrm{UI} e$ o controle recebeu placebo. Determinou-se parâmetros como: fatores de risco cardiometabólicos, resistência à insulina e valor contínuo da síndrome metabólica (cMetS). Neste estudo, não se identificou diferenças estatisticamente significantes entre parâmetros que pudessem interferir nos resultados e se chegou à conclusão de que aqueles que receberam Vitamina $D$ tiveram concentrações de insulina e triglicerídeos séricos reduzidos, assim como uma menor resistência insulínica e valor contínuo da síndrome metabólica, o que suporta o uso de Vitamina $D$ para atenuar sintomas diabéticos e melhorar o perfil cardiometabólico em crianças obesas.

Ryu $\mathrm{OH}$, et al. (2014) realizaram um estudo com 158 pacientes com DM2 que tinham controle glicêmico estável [hemoglobina A1c (HbA1c) $<8,5 \%$ ] e Vitamina D a níveis inferiores a $20 \mathrm{ng} / \mathrm{mL}$. Os participantes foram randomizados em dois grupos: Placebo (100 mg por dia de terapia cálcio administrado duas vezes ao dia) ou Vitamina D (1000 UI diárias de colecalciferol combinado com $100 \mathrm{mg}$ de cálcio administrado duas vezes por dia). Comparou-se atividade física ao ar livre, controle glicêmico, avaliação de homeostase - resistência à insulina (HOMA-IR) e paratormônio (PTH) durante a intervenção de 24 semanas. Foram analisados dados de 129 participantes (placebo $=65$, Vitamina $D=64$ ) e, embora houvesse diferenças significativas nos níveis de Vitamina D, não houve efeito no controle glicêmico em longo prazo para o DM2 em seus estudos.

Esses achados corroboram com os estudos de Nasri H, et al. (2014), Sheth JJ, et al. (2015) e Al-Shoumer $\mathrm{K}$ e Al-Essa $\mathrm{T}$ (2015), que fizerem estudos semelhantes e chegaram à conclusão que a suplementação de Vitamina D não interfere no controle do índice glicêmico. Entretanto, pelo fato de que pessoas com índices glicêmicos altos apresentarem baixos níveis de Vitamina D. Iqbal K, et al. (2016) acreditam que a associação entre deficiência de Vitamina $\mathrm{D}$ e HbA1C anormal em pacientes diabéticos paquistaneses e crianças com DMT1 (SAVASTIO S, et al., 2016) é sugestiva de que pacientes com Hipovitaminose D poderiam se beneficiar com a suplementação de Vitamina D (FONDJO LA, et al., 2017). 
Outro estudo utilizando perfis antropométricos e laboratoriais determinados em pacientes com um ou mais dos seguintes fatores de risco: hipertensão, índice de massa corporal (IMC) $\geq 25 \mathrm{~kg} / \mathrm{m} 2$, circunferência da cintura $>80 \mathrm{~cm}$ para mulheres e $>94 \mathrm{~cm}$ para homens, parentes de primeiro grau com diabetes, mulheres com bebês grandes para a idade gestacional ou recém-nascidos e com DM2 gestacional, Colesterol HDL (lipoproteína de alta densidade) $<35 \mathrm{mg} / \mathrm{dl}$ e triglicerídeos $>250 \mathrm{mg} / \mathrm{dl}$. Os pacientes foram divididos em dois grupos: um com pré-diabetes (jejum anormal, glicose no plasma ou teste oral de tolerância à glicose) e o outro com glicose normal (euglicêmica). Os resultados não mostraram diferenças estatísticas significantes entre o grupo pré-diabético $(n=38)$ e euglicêmico $(n=15)$ quanto à idade $(66,4 \pm 10,6$ versus $62,6 \pm 9,1$ anos), sexo (52,6 versus $73,3 \%$ feminino) e IMC (30,1 $\pm 4,61$ versus $27,9 \pm 4,7 \mathrm{~kg} / \mathrm{m} 2)$. Baixos níveis séricos de 25OHD3 foram encontrados em ambos grupos, sem diferenças estatísticas significativas entre eles $(29,1$ $\pm 11,8$ versus $26,87 \pm 9,2 \mathrm{ng} / \mathrm{dl}$ ). Os autores concluíram que não houve associação entre os níveis de 25OHD3 e as variáveis clínicas ou laboratoriais analisadas (GIORELLI GO, et al., 2015).

No diabetes, ocorre a acumulação de produtos avançados de glicação final (AGEs) e estes estão envolvidos na morbidez e mortalidade cardiovascular. Pesquisas feitas por Sebeková K, et al. (2015) revelaram que, em diabéticos, a Hipovitaminose $D$ não aumenta o acúmulo de AGEs, mas sim os marcadores de microinflamação e oxidação. A suplementação direcionada de Vitamina D por 16 semanas resultou em uma melhora pequena, mas não significativa, na função endotelial em grupos de indivíduos com diabetes mellitus tipo 2 (DALAN R, et al., 2016).

Adipocinas, como adiponectina, leptina e resistina são mediadores bioativos secretados pela adipose tecidual (HARINARAYAN CV, 2014). Eles têm um papel significativo na regulação do metabolismo de lipídios e carboidratos e envolvimento nas respostas inflamatórias e no DM2. De fato, alterações nos níveis circulantes de adipocinas em DM1 indicam envolvimento do tecido adiposo na patogênese do DM (GEYIKLI I, et al., 2013). De fato, alterações nos níveis circulantes de adipocinas em DM1 indicam envolvimento do tecido adiposo na patogênese do DM. Estudos revelaram que a Vitamina $D$ pode estar envolvida na regulação da resistina por meio de um mecanismo desconhecido (ISMAIL MM, et al., 2017). Além disso, Ding YH, et al. (2017) concluíram, por intermédio de estudos em chineses, que existe uma alta prevalência de Hipovitaminose $\mathrm{D}$ em pacientes com DM2. Além do mais, eles encontraram uma associação significativa entre a baixa concentração de soro $25(\mathrm{OH})$ D3 e a existência de alto índice de massa corporal e LDL (lipoproteína de Baixa Densidade) circulante alto, sendo fatores predisponentes para o aparecimento de placa aterosclerótica carotídea (PC) nesses pacientes com DM2.

$O$ baixo índice de Vitamina $D$ também está associado à geração e ao desenvolvimento de neuropatia periférica diabética (DPN) em caucasianos com DM2. Os pacientes asiáticos diabéticos com deficiência de Vitamina D são 1,22 vezes mais acometidos por DPN do que os com níveis normais de Vitamina $D$, sugerindo que suplementação de Vitamina $D$ é urgentemente necessária para prevenir o desenvolvimento de DPN no DM2 (GUANG-BO QU, et al., 2017).

\section{CONSIDERAÇÕES FINAIS}

Assim, pode-se concluir que a deficiência de Vitamina $D$ é um achado constante em pacientes que apresentam diabetes mellitus e que essa relação é inversamente proporcional ao tempo e ao agravamento da doença. Vários trabalhos demostram que a suplementação com Vitamina $D$ é uma ação necessária não só para os diabéticos, uma vez que, em pessoas saudáveis, foram encontrados baixos níveis da Vitamina $D$, pois ficou provado que a prevalência de Hipovitaminose $D$ está relacionada com o surgimento não só de complicações diabéticas, mas também com riscos para o sistema imunológico e cardiovascular.

\section{REFERÊNCIAS}

1. ALCUBLERRE $N$, et al. Vitamin D deficiency is associated with the presence and severity of diabetic retinopathy in type 2 diabetes mellitus. J. Diab. Res, 2015. Article ID 374178: 1-7.

2. ALHUMAIDI M, et al. Vitamin D deficiency in patients with type -2 diabetes mellitus in southern region of Saudi Arabia. Maedica, 2013. 8(3): 231-236.

3. AL-SHOUMER K, AL-ESSA T. Is there a relationship between vitamin D with insulin resistance and diabetes mellitus? World J. Diabetes, 2015. 6(8): 1057-1064.

4. BOILLAT-BLANCO N, et al. Association between tuberculosis, diabetes and 25 hydroxyvitamin D in Tanzania: a longitudinal case control study. BMC Infect. Dis, 2016. 16(1): 626. 
5. CORNACINI M, et al. Níveis séricos de vitamina D3 em mulheres com diabetes mellitus tipo 2. J. Health Sci. Inst, 2015. 33(1): 69-72.

6. DALAN R, et al. A randomised controlled trial evaluating the impact of targeted vitamin $\mathrm{D}$ supplementation on endothelial function in type 2 diabetes mellitus: the DIMENSION trial. Diab. Vasc. Dis. Res, 2016. 13(3): 192-200.

7. DHIA A, ALI A. Serum 25(OH) D in diabetes mellitus type 2: relation to glycaemic control. J. Clin. Diag. Res, 2013. 7(12): 2686-2688.

8. DING YH, et al. Association between serum 25-hydroxyvitamin D and carotid atherosclerotic plaque in Chinese type 2 diabetic patients. Medicine, 2017. 96(13): 6445.

9. FONDJO LA, et al. Vitamin D status and its association with insulin resistance among type 2 diabetics: a case-control study in Ghana. PLOS One, 2017. 14(2): 1-14.

10. GEDIK A, AKALIN S. Effects of Vitamin D deficiency and repletion on insulin and glucagons secretion in man. Diabetol, 1986. 29: 142-145.

11. GEYIKLI I, et al Increased resistin serum concentrations in patients with type 1 diabetes mellitus. J. Clin. Res. Pediatr. Endocrinol, 2013. 5(3): 189-193.

12. GIORELLI GO, et al. No association between 25-hydroxyvitamin D levels and prediabetes in Brazilian patients. A crosssectional study. Med J, 2015. 133(2): 73-77.

13. GRIZ LH, et al. Vitamin D and diabetes mellitus: an update - 2013. Arq. Bras. Endocrinol. Metab, 2014. 58(1): 1-8.

14. HAIDARI F, et al. Association between serum vitamin $D$ level and glycemic and inflammatory markers in non-obese patients with type 2 diabetes. Iran J. Med Sci, 2016. 41(5): 367-373.

15. HARINARAYAN CV. Vitamin D and diabetes mellitus. Hormones, 2014. 13(2): 163-81.

16. QBAL K, et al. Association of vitamin D deficiency with poor glycaemic control in diabetic patients. J. Pak. Med. Assoc, 2016. 66(12): 1562-1565.

17. ISMAIL MM, et al. Serum adipokines and vitamin D levels in patients with type 1 diabetes mellitus. Arch. Med. Sci, 2017. 13(4): 738-744.

18. KELISHADI R, et al. Effects of vitamin D supplementation on insulin resistance and cardiometabolic risk factors in children with metabolic syndrome: a triple-masked controlled trial. J. Pediatr, 2014. 90(1): 28-34.

19. KIMBALL S, et al. Vitamin D: a growing perspective. Crit. Rev. Clin. Lab. Sci, 2008. 45(4): 339-414.

20. KOSTOGLOU-ATHANASSIOU I, et al. Vitamin D and glycemic control in diabetes mellitus type 2. Ther. Adv. Endocrinol. Metab, 2013. 4(4): 122-128.

21. LICHTENSTEIN A, et al. Vitamina D: ações sextraósseas e uso racional. Rev. Assoc. Med. Bras, 2013. 59(5): 495506.

22. LUO B, et al. The association between vitamin $D$ deficiency and diabetic retinopathy in type 2 diabetes: a meta-analysis of observational studies. Nutrients, 2017. 9(3): 307.

23. MACHADO MRC, et al. Vitamina $D$ e diabetes mellitus, suas epidemias e o envelhecimento. O que há de novo? Reprod. Clim, 2014. 29(2): 54-59.

24. MARQUES CDL, et al. A importância dos níveis de vitamina D nas doenças autoimunes. Rev. Bras. Reumatol, 2010. 50(1): 67-80.

25. MUSZKAT P, et al. Evidence-based non-skeletal actions of vitamin D. Arq. Bras. Endocrinol. Metab, 2010. 54(2): 110117.

26. NASRI H, et al. Efficacy of supplementary vitamin D on improvement of glycemic parameters in patients with type 2 diabetes mellitus; a randomized double blind clinical trial. J. Renal Inj. Prev, 2014. 3(1): 31-34.

27. OLIVEIRA KC, ZANETTI ML. Conhecimento e atitude de usuários com diabetes mellitus em um Serviço de Atenção Básica à Saúde. Rev. Esc. Enferm. USP, 2011. 45(4): 862-868.

28. OLIVEIRA RC. Diabetes mellitus tipo 2 e o seu impacto para o estilo de vida dos portadores. Monografia (Graduação em Enfermagem). Universidade Federal da Paraíba, João Pessoa, 2006; 40p.

29. OLIVERI B, ZENI S. Hipovitaminosis D y desarrollo de Síndrome Metabólico. Acta Bioquím. Clín. Latinoam, 2016. 50(3): 387-393.

30. PAN GT, et al. Vitamin D deficiency in relation to the risk of metabolic syndrome in middle-aged and elderly patients with type 2 diabetes mellitus. J. Nutr. Sci. Vitaminol, 2016. 62(4): 213-219.

31. PARISI MCR. Úlceras no pé diabético. In: Jorge AS, Dantas SRPE. Abordagem multiprofissional ao tratamento de feridas. São Paulo: Atheneu, 2003. p. 311-317.

32. QU GB, et al. The association between vitamin D level and diabetic peripheral neuropathy in patients with type 2 diabetes mellitus: An update systematic review and meta-analysis. J. Clin. Transl. Endocrinol, 2017. 9: 25-31.

33. RAFAELLI RA, et al. Influência da vitamina D nas doenças endocrinometabólicas. Semina: Ci. Biol. Saúde, 2015. 36(1): 333-348.

34. $\mathrm{RYU} \mathrm{OH}$, et al. A prospective randomized controlled trial of the effects of vitamin $\mathrm{D}$ supplementation on long-term glycemic control in type 2 diabetes mellitus of Korea. Endocr, 2014. J. 61(2): 167-176.

35. SAVASTIO S, et al. Vitamin D deficiency and glycemic status in children and adolescents with type 1 diabetes mellitus. PLoS One, 2016. 11(9): 1-13.

36. SCHUCH NJ, et al. Vitamina D e doenças endocrinometabólicas. Arq. Bras. Endocrinol. Metab, 2009. 53(5): 625-633.

37. SEBEKOVÁ K, et al. Is vitamin D deficiency related to accumulation of advanced glycation end products, markers of inflammation, and oxidative stress in diabetic subjects?. Biomed Res. Int, 2015. 2015: 1-15.

38. SHETH JJ, et al. Does vitamin D play a significant role in type 2 diabetes?. BMC Endocrine Disorders, 2015. 15(5): E307.

39. THACHER TD, CLARKE BL. Vitamin D insufficiency. Clin. Proc, 2011. 86(1): 50-60.

40. WORLD HEALTH ORGANIZATION, 2011. In: Diabetes: diabetes facts. Fact sheet. 312. France: NMH. 